\title{
Comportamentos de risco à saúde em adolescentes no Sul do Brasil: prevalência e fatores associados
}

\author{
José Cazuza de Farias Júnior, ${ }^{1}$ Markus Vinicius Nahas, ${ }^{2}$ \\ Mauro Virgílio Gomes de Barros, ${ }^{3}$ Mathias Roberto Loch, ${ }^{4}$ \\ Elusa Santina A. de Oliveira, ${ }^{2}$ Maria Fermínia Luchtemberg De Bem² \\ e Adair da Silva Lopes ${ }^{2}$
}

Como citar Farias Júnior JC, Nahas MV, Barros MVG, Loch MR, Oliveira ESA, De Bem MFL, Lopes AS. Comportamentos de risco à saúde em adolescentes no Sul do Brasil: prevalência e fatores associados. Rev Panam Salud Publica. 2009;25(4):344-52.

RESUMO Objetivo. Determinar a prevalência de comportamentos de risco à saúde e analisar fatores associados à exposição a esses comportamentos em adolescentes do Estado de Santa Catarina, Brasil.

Método. Adolescentes escolares de 240 turmas do ensino médio da rede pública estadual responderam a um questionário para a coleta de informações demográficas (sexo, idade, região de residência e período de estudo), socioeconômicas (trabalho e renda familiar mensal) e de comportamentos de risco à saúde (níveis insuficientes de atividade física, baixo consumo de frutas/verduras, tabagismo, consumo abusivo de bebidas alcoólicas, consumo de drogas ilícitas, envolvimento em brigas e não utilizar preservativos regularmente nas relações sexuais).

Resultados. Foram considerados válidos os questionários de 5028 adolescentes (2 984 do sexo feminino e 2044 do sexo masculino), com idade entre 15 e 19 anos (média = 17,0; desvio padrão $=1,12$ ). Os comportamentos de risco mais prevalentes na amostra foram níveis insuficientes de atividade física (36,5\%), baixo consumo de frutas/verduras $(46,5 \%)$ e não utilizar preservativos regularmente nas relações sexuais (38,3\%). Aproximadamente sete em cada 10 adolescentes $(64,7 \%)$ estavam expostos a dois ou mais comportamentos de risco de forma simultânea. Os seguintes subgrupos de risco foram identificados: adolescentes do sexo masculino, adolescentes de 18 a 19 anos de idade, que residem em área urbana, que estudam no período noturno e que têm maior renda familiar.

Conclusões. A proporção de adolescentes expostos a comportamentos de risco à saúde foi elevada, principalmente ao se considerar a exposição simultânea a diferentes comportamentos. Os resultados encontrados podem contribuir para o desenvolvimento de programas de promoção da saúde no ambiente escolar, direcionados principalmente aos subgrupos de risco.

Palavras-chave Adolescente, comportamento de risco, escolares, estilo de vida, saúde, Brasil.

Universidade Federal da Paraíba (UFPB), Departamento de Educação Física, Centro de Ciências da Saúde. Universidade Federal de Santa Catarina (UFSC), Centro de Desportos, Programa de PósGraduação em Educação Física e Núcleo de Pesquisa em Atividade Física e Saúde (NuPAF). Bolsista CAPES. Correspondência: Universidade Federal de Santa Catarina, Centro de Desportos, Campus Universitário - Trindade, CEP 88049-900, Florianópolis, SC, Brasil. E-mail: jcazuzajr@hotmail.com
2 Universidade Federal de Santa Catarina (UFSC), Centro de Desportos, Programa de Pós-Graduação em Educação Física e Núcleo de Pesquisa em Atividade Física e Saúde (NuPAF).

3 Universidade de Pernambuco (UPE), Grupo de Pesquisa em Estilos de Vida e Saúde.

4 Universidade Estadual de Londrina (UEL), Departamento de Educação Física.
A influência do estilo de vida sobre os níveis de saúde e qualidade de vida de diferentes grupos populacionais, incluindo crianças e adolescentes, tem sido amplamente documentada na literatura na área da saúde $(1,2)$. Nesse sentido, o levantamento, o monitoramento e a intervenção sobre comportamentos de 
risco à saúde têm sido considerados por diversas agências de saúde como prioridades de saúde pública $(3,4)$. Entre os comportamentos de risco, é possível citar níveis insuficientes de atividade física, hábitos alimentares inadequados, tabagismo, consumo abusivo de álcool, consumo de drogas ilícitas, envolvimento em situações de violência e comportamentos sexuais de risco $(3,4)$.

Diversos problemas de saúde têm sido associados à exposição a comportamentos de risco, tanto na população jovem (mortes por causas violentas e por acidentes de veículo a motor, doenças sexualmente transmissíveis, gravidez precoce) quanto em adultos em idade potencialmente produtiva (doenças crônicas não-transmissíveis) (4-6). Além disso, uma grande parcela da população jovem está exposta a um ou mais comportamentos de risco (7), os quais podem estar inter-relacionados (8) e se estender à idade adulta (9). Por exemplo, tem-se observado que alguns comportamentos de risco (níveis insuficientes de atividade física, tabagismo e consumo abusivo de bebidas alcoólicas) medidos na infância e adolescência estão associados a fatores de risco para doença cardiovascular e à presença de doenças cardiovasculares na fase adulta (10).

No Brasil, estudos sobre comportamentos de risco à saúde em adolescentes foram desenvolvidos com amostras ou muito específicas (11) ou restritas a uma determinada cidade (12-17). Além disso, a ocorrência simultânea de comportamentos de risco praticamente não foi explorada nesses estudos.

Informações sobre a prevalência de comportamentos de risco à saúde e os fatores associados à exposição a esses comportamentos podem contribuir para a identificação de grupos de risco, para o monitoramento dos níveis de saúde da população jovem e para subsidiar o desenvolvimento de políticas e programas de promoção da saúde. Nesse sentido, os objetivos deste estudo foram determinar a prevalência de comportamentos de risco à saúde de forma isolada e simultânea em adolescentes no sul do Brasil e analisar os fatores associados à exposição a esses comportamentos de risco.

\section{MATERIAIS E MÉTODOS}

Santa Catarina é um estado da Região Sul do Brasil, com população de 5,4 milhões de habitantes (18), em sua maio- ria de origem européia (principalmente alemã, italiana e portuguesa), com renda per capita de 12 mil reais (quinta maior do país) e o quarto melhor índice de desenvolvimento humano do país (IDH $=0,82$, ano-base 2000). Nesse período, a população de adolescentes de 15 a 19 anos, foco deste estudo, era de $535 \mathrm{mil}$.

O presente estudo foi desenvolvido mediante análise secundária dos dados de um levantamento epidemiológico transversal, de base escolar, denominado Comportamento do Adolescente Catarinense (COMPAC), realizado pelo Núcleo de Pesquisa em Atividade Física e Saúde (NuPAF) da Universidade Federal de Santa Catarina (UFSC), em $2001 .^{5}$ O protocolo de investigação foi aprovado pelo Comitê de Ética em Pesquisa da UFSC sob o número $064 / 2000$.

$\mathrm{Na}$ época, dados oficiais da Secretaria de Estado da Educação indicavam um total de 205 mil estudantes nas 598 escolas públicas de ensino médio no Estado de Santa Catarina, localizadas nas zonas rural e urbana, distribuídas em 26 Coordenadorias Regionais de Educação (CRE) nas seis regiões geográficas que compreendem o estado (Oeste, Planalto Serrano, Norte, Vale do Itajaí, Litoral e Sul) (19).

O processo de amostragem foi conduzido em dois estágios. No estágio inicial, foram selecionadas 172 escolas estaduais, de forma aleatória e proporcional, considerando-se a região geográfica, a CRE e o porte da escola (pequena: $<200$ alunos; média: 200 a 499 alunos; grande: $>500$ alunos). No segundo estágio, foram selecionadas, de forma aleatória e proporcional (considerando-se o turno e as séries do ensino médio), 240 turmas dentre as 6094 existentes. A partir de informações da Secretaria de Estado da Educação, considerou-se que essas turmas tinham, em média, 25 alunos.

$\mathrm{Na}$ determinação do tamanho da amostra, em função dos múltiplos comportamentos de risco investigados no estudo, considerou-se uma prevalência de $50 \%$ como a maior esperada, com intervalo de confiança de 95\% (IC95\%) e erro de 2 pontos percentuais. Dados esses parâmetros, o tamanho mínimo da

\footnotetext{
Nahas MV, Barros MVG, De Bem MFL, Oliveira ESA, Loch MR. Estilo de vida e indicadores de saúde dos jovens catarinenses. Relatório da pesquisa desenvolvida pelo Núcleo de Pesquisa em Atividade Física e Saúde com amostra representativa dos estudantes de 15 a 19 anos matriculados nas escolas estaduais do ensino médio em Santa Catarina. Florianópolis: Universidade $\mathrm{Fe}-$ deral de Santa Catarina; 2005.
}

amostra seria de 2395 adolescentes escolares, número que foi multiplicado por 2 em função do efeito de delineamento $($ deff $=2)$, por se tratar de um processo amostral por conglomerado. Houve um acréscimo de $25 \%$ no tamanho da amostra para compensar possíveis perdas e recusas. A amostra final foi definida em 5988 sujeitos, e o questionário foi aplicado a 5463 escolares do ensino médio.

Para o estudo dos fatores associados aos comportamentos de risco à saúde, a amostra permitiu detectar como significativas razões de prevalência de 1,15 ou mais para exposições afetando de 5 a $75 \%$ da população estudada, com $80 \%$ de poder e $95 \%$ de nível de confiança.

$\mathrm{Na}$ coleta de dados, utilizou-se o Questionário sobre Estilo de Vida e Comportamentos de Risco de Adolescentes Catarinenses empregado no estudo COMPAC, desenvolvido com base em partes de instrumentos já validados para adolescentes, que investiga os seguintes comportamentos de risco à saúde: níveis insuficientes de atividade física, baixo consumo de frutas/verduras, tabagismo, consumo abusivo de bebidas alcoólicas, envolvimento em brigas, consumo de drogas ilícitas e a não-utilização regular de preservativos nas relações sexuais. Previamente à aplicação do instrumento, os escolares foram informados sobre os objetivos da pesquisa e sobre a preservação do anonimato. O questionário foi aplicado em cada sala de aula selecionada (grupos de 20 a 30 alunos) por equipe treinada e sob a supervisão dos pesquisadores responsáveis pelo projeto. Após o preenchimento, que levava entre 30 e 40 minutos, os escolares depositavam os questionários em uma caixa sem identificação.

Todos os escolares das turmas sorteadas presentes no dia da coleta e que tinham entre 15 e 19 anos responderam ao questionário, mediante autorização prévia. As variáveis em estudo, as medidas utilizadas e as categorias adotadas na análise dos dados estão descritas na tabela 1 .

Os dados coletados no questionário foram digitados e analisados no programa Epi Info 6.04d com checagem automática de consistência e amplitude e erro de digitação de no máximo 1,26\%. Todos os erros identificados foram corrigidos, excluindo-se os outliers.

O teste do qui-quadrado $\left(\chi^{2}\right)$ foi utilizado para avaliar a heterogeneidade dos indicadores demográficos e socioeconômicos em relação à prevalência de com- 
TABELA 1. Variáveis em estudo, medidas utilizadas e categorias adotadas na análise dos dados, Estado de Santa Catarina, Brasil, 2001

\begin{tabular}{|c|c|c|}
\hline Variáveis & Medidas (autorreferidas) & Categorias \\
\hline $\begin{array}{l}\text { Variáveis demográficas } \\
\text { Sexo }\end{array}$ & & $\begin{array}{l}\text { Feminino }=0 \\
\text { Masculino }=1\end{array}$ \\
\hline Idade & $\begin{array}{l}\text { Diferença entre o ano da coleta de dados e o ano de nascimento, dos } 15 \\
\text { aos } 19 \text { anos, agrupando-se os dados da seguinte forma: } 15 \text { a } 15,99=15 \\
\text { anos, etc. }\end{array}$ & $\begin{array}{l}15 \text { a } 17 \text { anos }=0 \\
18 \text { a } 19 \text { anos }=1\end{array}$ \\
\hline Região de residência & Região da cidade em que morava & $\begin{array}{l}\text { Zona rural }=0 \\
\text { Zona urbana }=1\end{array}$ \\
\hline Período de estudo & Período em que estava matriculado na escola & $\begin{array}{l}\text { Diurno }=0 \\
\text { Noturno }=1\end{array}$ \\
\hline $\begin{array}{l}\text { Variáveis socioeconômicas } \\
\text { Trabalho }\end{array}$ & $\begin{array}{l}\text { Trabalhar independentemente de ser remunerado; não inclui trabalho } \\
\text { doméstico }\end{array}$ & $\begin{array}{l}\text { Não }=0 \\
\text { Sim }=1\end{array}$ \\
\hline Renda familiar mensal & Soma de todos os rendimentos mensais de todos os membros da família & $\begin{array}{l}>\mathrm{R} \$ 1000 / \text { mês }=0 \\
\leq \mathrm{R} \$ 1000 / \mathrm{mês}=1\end{array}$ \\
\hline \multicolumn{3}{|l|}{ Comportamentos } \\
\hline Atividade física & $\begin{array}{l}\text { Frequência semanal (dias por semana) e tempo de prática (minutos por } \\
\text { dia) de atividades físicas moderadas/vigorosas: esportes, atividades de } \\
\text { lazer, atividades domésticas, deslocamento ativo (caminhar, pedalar) }\end{array}$ & $\begin{array}{l}\geq 300 \mathrm{~min} / \mathrm{semana}=0 \\
<300 \mathrm{~min} / \mathrm{semana}=1^{\mathrm{a}}\end{array}$ \\
\hline $\begin{array}{l}\text { Hábitos alimentares } \\
\text { (consumo de } \\
\text { frutas/verduras) }\end{array}$ & $\begin{array}{l}\text { Frequência de consumo de frutas e verduras durante uma semana típica } \\
\text { (dias por semana) }\end{array}$ & $\begin{array}{l}\geq 4 \text { dias } / \text { semana }=0 \\
<4 \text { dias } / \text { semana }=1^{b}\end{array}$ \\
\hline Tabagismo & $\begin{array}{l}\text { Consumir fumo pelo menos uma vez por semana durante uma semana } \\
\text { típica }\end{array}$ & $\begin{array}{l}\text { Não }=0 \\
\text { Sim }=1^{c}\end{array}$ \\
\hline Bebidas alcoólicas & $\begin{array}{l}\text { Consumo abusivo de bebidas alcoólicas durante uma semana típica } \\
\text { (número de dias por semana e doses consumidas por semana) }\end{array}$ & $\begin{array}{l}\text { Feminino: } \\
<7 \text { doses } / \text { semana }=0 \\
\geq 7 \text { doses } / \text { semana }=1^{d} \\
\text { Masculino: } \\
<14 \text { doses } / \text { semana }=0 \\
\geq 14 \text { doses } / \text { semana }=1^{d}\end{array}$ \\
\hline Drogas ilícitas & Consumo de drogas ilícitas (excluindo fumo e álcool) & $\begin{array}{l}\text { Não }=0 \\
\text { Sim }=1^{e}\end{array}$ \\
\hline Brigas (lutas) & $\begin{array}{l}\text { Envolvimento em brigas (lutas) nos } 12 \text { meses que antecederam a coleta } \\
\text { de dados }\end{array}$ & $\begin{array}{l}\leq 1 \mathrm{vez}=0 \\
>1 \mathrm{vez}=1^{f}\end{array}$ \\
\hline Uso de preservativos & $\begin{array}{l}\text { Frequência de uso de preservativos nas relações sexuais (entre os que } \\
\text { tinham vida sexualmente ativa) }\end{array}$ & $\begin{array}{l}\text { Sempre }=0 \\
\text { Raramente/nunca }=1^{\mathrm{g}}\end{array}$ \\
\hline
\end{tabular}

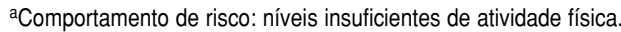

${ }^{b}$ Comportamento de risco: baixo consumo de frutas/verduras.

${ }^{c}$ Comportamento de risco: tabagismo.

${ }^{\mathrm{d}}$ Comportamento de risco: consumo abusivo de bebidas alcoólicas.

e Comportamento de risco: consumo de drogas ilícitas.

${ }^{\dagger}$ Comportamento de risco: envolvimento em brigas.

${ }^{g}$ Comportamento de risco: não utilizar preservativos regularmente nas relações sexuais.

portamentos de risco à saúde de forma isolada e simultânea. Para avaliar a associação bruta e ajustada entre as variáveis demográficas e socioeconômicas e a presença isolada e simultânea de comportamentos de risco à saúde, utilizou-se a medida de razão de prevalência (RP), com respectivos IC95\%, a partir da regressão de Poisson com variância robusta.

$\mathrm{Na}$ análise isolada dos fatores associados aos comportamentos de risco, cada comportamento de risco foi considerado como variável dependente (desfecho), e os indicadores demográficos e socioeconômicos, como variáveis independen- tes (exposição). Para a análise da associação entre exposição simultânea a diferentes comportamentos de risco e indicadores demográficos e socioeconômicos, foram considerados três modelos: no primeiro modelo, buscou-se identificar as variáveis associadas à exposição a um ou mais comportamentos de risco à saúde versus aqueles que não apresentavam nenhum comportamento de risco; no segundo e terceiro modelos, respectivamente, foram considerados como variáveis dependentes a exposição a dois ou mais e três ou mais comportamentos de risco à saúde de forma simultânea, man- tendo-se os indicadores demográficos e socioeconômicos como variáveis independentes. Nas análises ajustadas, todas as variáveis dependentes foram incluídas no modelo em um mesmo nível de determinação.

Todos os procedimentos de análise estatística foram realizados no programa Stata 10.0, adotando-se um nível de significância de 5\%.

\section{RESULTADOS}

O questionário foi respondido por 5463 escolares do ensino médio, sendo 
excluídos os que não completariam 15 anos no ano da coleta ou que já haviam completado 20 anos $(n=378,6,9 \%)$ e os que entregaram o questionário com muitas questões sem resposta $(n=57,1,1 \%)$, resultando em uma amostra final de 5028 adolescentes (2 984 moças e 2044 rapazes) com idade entre 15 e 19 anos (média $=17,0$, desvio padrão $=1,12$ ). A taxa de valores ignorados (itens não respondidos) não ultrapassou $15 \%$ para as variáveis de desfecho (comportamentos de risco) e foi de 1,8\% para as variáveis de exposição (indicadores demográficos e socioeconômicos). Outras informações sobre as características da amostra estão descritas na tabela 2 .

Níveis insuficientes de atividade física $(36,5 \%)$, baixo consumo de frutas/verduras $(46,5 \%)$ e não utilizar preservativos regularmente nas relações sexuais $(38,3 \%)$ foram os comportamentos de risco mais prevalentes nos jovens da amostra (tabela 3).

Adolescentes do sexo masculino apresentaram maior prevalência de baixo consumo de frutas/verduras, consumo

TABELA 2. Características demográficas e socioeconômicas dos adolescentes escolares do ensino médio do Estado de Santa Catarina, Brasil, 2001

\begin{tabular}{|c|c|c|c|c|c|c|}
\hline \multirow[b]{2}{*}{ Variável } & \multicolumn{2}{|c|}{ Todos } & \multicolumn{2}{|c|}{ Masculino } & \multicolumn{2}{|c|}{ Feminino } \\
\hline & No. & $\%$ & No. & $\%$ & No. & $\%$ \\
\hline \multicolumn{7}{|l|}{ Idade (anos) } \\
\hline 15 a 17 & 3961 & 78,8 & 1542 & 75,4 & 2419 & 81,1 \\
\hline 18 a 19 & 1067 & 21,2 & 502 & 24,6 & 565 & 18,9 \\
\hline \multicolumn{7}{|l|}{ Região de residência } \\
\hline Rural & 1019 & 20,4 & 466 & 23,0 & 553 & 18,6 \\
\hline Urbana & 3981 & 79,6 & 1562 & 77,0 & 2419 & 81,4 \\
\hline \multicolumn{7}{|l|}{ Período de estudo } \\
\hline Diurno (manhã/tarde) & 2202 & 43,8 & 804 & 39,3 & 1398 & 46,9 \\
\hline Noturno & 2825 & 56,2 & 1240 & 60,7 & 1585 & 53,1 \\
\hline \multicolumn{7}{|l|}{ Trabalho } \\
\hline Sim & 2733 & 54,9 & 1275 & 63,0 & 1458 & 49,4 \\
\hline Não & 2242 & 45,1 & 748 & 37,0 & 1494 & 50,6 \\
\hline \multicolumn{7}{|l|}{ Renda familiar mensala } \\
\hline$\leq \mathrm{R} \$ 1000$ & 1404 & 28,5 & 1360 & 67,8 & 757 & 25,9 \\
\hline$>\mathrm{R} \$ 1000$ & 3529 & 71,5 & 647 & 32,2 & 2169 & 74,1 \\
\hline
\end{tabular}

a Valor do salário mínimo em 2001: $\mathrm{R} \$ 180,00$.

abusivo de bebidas alcoólicas, consumo de drogas ilícitas e envolvimento em brigas. As jovens do sexo feminino, por sua vez, apresentaram maior prevalência de níveis insuficientes de atividade física e comportamento sexual de risco (não uti- lizar preservativos regularmente nas relações sexuais). O tabagismo não apresentou diferenças significativas entre os sexos (tabela 3).

Nos adolescentes mais velhos (18 a 19 anos), a prevalência de níveis insuficien-

TABELA 3. Prevalência e razão de prevalência bruta para comportamentos de risco à saúde em adolescentes escolares do ensino médio do Estado de Santa Catarina, Brasil, 2001

\begin{tabular}{|c|c|c|c|c|c|c|c|}
\hline Variávela $^{a}$ & $\begin{array}{c}\text { Níveis } \\
\text { insuficientes } \\
\text { de atividade } \\
\text { física } \\
\% \text { (no.) }\end{array}$ & $\begin{array}{c}\text { Baixo } \\
\text { consumo de } \\
\text { frutas/verdures } \\
\%(\text { no.) }\end{array}$ & $\begin{array}{c}\text { Tabagismo } \\
\% \text { (no.) }\end{array}$ & $\begin{array}{l}\text { Consumo } \\
\text { abusivo de } \\
\text { bebidas } \\
\text { alcoólicas } \\
\% \text { (no.) }\end{array}$ & $\begin{array}{c}\text { Consumo } \\
\text { de drogas } \\
\text { ilícitas } \\
\%(\text { no.) }\end{array}$ & $\begin{array}{c}\text { Envolvimento } \\
\text { em brigas } \\
\% \text { (no.) }\end{array}$ & $\begin{array}{l}\text { Não utilizar } \\
\text { preservativos } \\
\text { com } \\
\text { regularidade } \\
\% \text { (no.) }\end{array}$ \\
\hline Todos & 36,5 (1 782) & 46,5 (2 290) & $6,8(336)$ & 27,4 (1 193) & $10,4(496)$ & $16,5(771)$ & $38,3(825)$ \\
\hline \multicolumn{8}{|l|}{ Sexo } \\
\hline Femininob & $43,7(1260)^{c}$ & 42,4 (1 243) & $6,5(192)$ & $20,7(529)$ & $8,1(231)$ & $10,4(283)$ & $47,0(504)^{c}$ \\
\hline Masculino & $26,1(522)$ & $52,5(1047)^{\mathrm{C}}$ & $7,2(144)$ & $36,8(665)^{c}$ & $13,7(265)^{c}$ & $24,9(488)^{\mathrm{c}}$ & $29,7(321)$ \\
\hline$R P(I C 95 \%)^{d}$ & $0,59(0,53$ a 0,66$)$ & $1,23(1,14$ a 1,34$)$ & $1,10(0,88$ a 1,36$)$ & $1,77(1,58$ a 1,99$)$ & $1,70(1,42$ a 2,02$)$ & $2,38(2,06$ a 2,76$)$ & $0,63(0,54$ a 0,72$)$ \\
\hline \multicolumn{8}{|l|}{ Idade (anos) } \\
\hline 15 a $17^{b}$ & $35,0(1356)$ & 46,3 (1 803) & $6,0(237)$ & $26,2(902)$ & $9,1(343)$ & $16,6(610)$ & $37,5(558)$ \\
\hline 18 a 19 & $42,2(426)^{c}$ & $47,1(487)$ & $9,4(99)^{c}$ & $31,7(292)^{c}$ & $14,9(153)^{c}$ & $16,1(161)$ & $40,3(267)$ \\
\hline RP (IC95\%) & $1,20(1,08$ a 1,34$)$ & $1,01(0,91$ a 1,12$)$ & $1,55(1,23$ a 1,97$)$ & $1,20(1,06$ a 1,38$)$ & $1,63(1,34$ a 1,97$)$ & $0,97(0,81$ a 1,15$)$ & $1,07(0,92$ a 1,24$)$ \\
\hline \multicolumn{8}{|c|}{ Região de residência } \\
\hline Rural ${ }^{b}$ & $36,3(357)$ & $43,2(429)$ & $3,5(35)$ & $29,2(253)$ & $4,5(43)$ & $14,8(139)$ & $33,0(115)$ \\
\hline Urbana & $36,4(1412)$ & $47,3(1848)^{c}$ & $7,6(300)^{c}$ & 26,0 (932) & $11,8(449)^{c}$ & $16,9(628)$ & $39,5(709)^{c}$ \\
\hline $\operatorname{RP}(\operatorname{IC} 95 \%)^{d}$ & $1,0(0,89$ a 1,12$)$ & $1,09(1,02$ a 1,21$)$ & $2,18(1,53$ a 3,09$)$ & $0,91(0,79$ a 1,05$)$ & $2,65(1,93$ a 3,62$)$ & $1,14(0,95$ a 1,37$)$ & $1,19(0,98$ a 1,45$)$ \\
\hline \multicolumn{8}{|c|}{ Período de estudo } \\
\hline Diurno ${ }^{b}$ & 30,0 (652) & $45,1(977)$ & $5,3(116)$ & $22,4(435)$ & $8,4(176)$ & $15,9(327)$ & $34,8(248)^{c}$ \\
\hline Noturno & $41,7(1129)^{c}$ & 47,5 (1 312) & 7,9 (220) & $31,4(759)^{c}$ & $11,9(320)^{c}$ & $17,0(444)$ & $40,1(577)$ \\
\hline $\operatorname{RP}\left(\right.$ IC95\%) ${ }^{d}$ & $1,38(1,26$ a 1,52$)$ & $1,05(0,97$ a 1,14$)$ & $1,48(1,18$ a 1,85$)$ & $1,40(1,24$ a 1,57$)$ & $1,41(1,17$ a 1,70$)$ & $1,06(0,92$ a 1,23$)$ & $1,15(1,01$ a 1,33$)$ \\
\hline \multicolumn{8}{|l|}{ Trabalho } \\
\hline Nãob & $34,3(751)$ & 44,5 (984) & $6,5(144)$ & $23,7(467)$ & $8,8(188)$ & $14,9(310)$ & 40,5 (332) \\
\hline Sim & $38,6(1021)^{c}$ & $48,2(1286)^{c}$ & $7,0(190)$ & $30,3(714)^{c}$ & $11,7(306)^{c}$ & $17,8(452)^{c}$ & $36,8(485)$ \\
\hline RP (IC95\%) $)^{d}$ & $1,19(1,02$ a 1,23$)$ & $1,08(1,02$ a 1,17$)$ & $1,08(0,87$ a 1,34$)$ & $1,27(1,13$ a 1,43$)$ & $1,32(1,10$ a 1,58$)$ & $1,18(1,02$ a 1,37$)$ & $0,90(0,78$ a 1,04$)$ \\
\hline \multicolumn{8}{|c|}{ Renda familiar mensal } \\
\hline$\leq \mathrm{R} \$ 1000^{\mathrm{b}}$ & $35,8(494)$ & 41,6 (574) & $7,8(108)$ & $30,4(378)^{c}$ & $13,2(177)^{c}$ & $18,6(244)^{c}$ & $36,4(247)$ \\
\hline$>\mathrm{R} \$ 1000$ & 36,7 (1 254) & $48,5(1680)^{c}$ & $6,3(221)$ & $26,2(797)$ & $9,3(311)$ & $15,9(520)$ & $39,3(567)$ \\
\hline$R P(I C 95 \%)^{d}$ & $1,02(0,92$ a 1,13$)$ & $1,16(1,05$ a 1,27$)$ & $0,81(0,64$ a 1,02$)$ & $0,86(0,76$ a 0,97$)$ & $0,70(0,58$ a 0,84$)$ & $0,85(0,73$ a 0,99$)$ & $1,07(0,92$ a 1,25$)$ \\
\hline
\end{tabular}

a Máximo de 1,8\% de valores ignorados para variáveis demográficas e socioeconômicas (exposições) e de 15\% para comportamentos de risco (desfechos).

${ }^{b}$ Categoria de referência.

c Teste para heterogeneidade, $p<0,01$.

${ }^{d}$ Razão de prevalência bruta e respectivo intervalo de confiança de $95 \%$. 
tes de atividade física, tabagismo, consumo abusivo de bebidas alcoólicas e de drogas ilícitas foi mais elevada do que nos seus pares mais jovens (15 a 17 anos). Os demais comportamentos de risco não apresentaram diferenças entre os grupos etários (tabela 3).

Ao se comparar adolescentes que residiam na região urbana e na rural, diferenças significativas foram observadas apenas com relação a baixo consumo de frutas/verduras, tabagismo e consumo de drogas ilícitas, que tiveram prevalência mais elevada nos adolescentes que residiam na região urbana (tabela 3).

Os adolescentes que estudavam no período noturno apresentaram maior prevalência de comportamentos de risco (inatividade física, consumo abusivo de bebidas alcoólicas, consumo de drogas ilícitas e não utilizar preservativos regularmente nas relações sexuais) em relação aos seus respectivos pares que estudavam no período diurno (manhã/ tarde); o mesmo foi observado entre os adolescentes que trabalhavam (inatividade física, baixo consumo de frutas/ verduras, consumo abusivo de bebidas alcoólicas, consumo de drogas ilícitas e envolvimento em brigas) versus os que não trabalhavam (tabela 3).

Adolescentes que pertenciam a famílias com maior renda familiar mensal (> R\$ $1000 /$ mês) apresentaram maior prevalência de baixo consumo de fru- tas/verduras; já os que pertenciam a famílias com menor renda familiar mensal ( $\leq \mathrm{R} \$ 1000 /$ mês) apresentaram maior prevalência de consumo abusivo de bebidas alcoólicas, consumo de drogas ilícitas e envolvimento em brigas (tabela 3).

Após o ajuste para as variáveis de cada modelo, foram identificados os seguintes subgrupos de risco: a) adolescentes do sexo masculino (baixo consumo de frutas/verduras, consumo abusivo de bebidas alcoólicas, consumo de drogas ilícitas e envolvimento em brigas); b) adolescentes de 18 a 19 anos de idade (níveis insuficientes de atividade física, tabagismo e consumo de drogas ilícitas); c) adolescentes que residiam em área urbana (baixo consumo de frutas/verduras, tabagismo e consumo de drogas ilícitas); d) adolescentes que estudavam no período noturno (níveis insuficientes de atividade física, tabagismo, consumo abusivo de bebidas alcoólicas e consumo de drogas ilícitas); e) adolescentes que tinham maior renda familiar (baixo consumo de frutas/verduras) (tabela 4).

Menos de $10 \%$ dos adolescentes não apresentaram comportamento de risco; um quarto deles apresentou um comportamento de risco, e aproximadamente sete em cada $10(64,7 \%)$ apresentaram dois ou mais comportamentos de risco à saúde simultaneamente (figura 1). Não foram observadas diferenças estatistica- mente significativas entre adolescentes do sexo masculino e feminino.

$\mathrm{Na}$ análise bruta (tabela 5), observouse que a exposição a comportamentos de risco de forma simultânea estava positivamente associada à idade, ao período de estudo e ao trabalho, ou seja, adolescentes mais velhos (18 a 19 anos), que estudavam no período noturno e que trabalhavam tinham maior probabilidade de exposição a três ou mais comportamentos de risco, simultaneamente, se comparados a adolescentes mais jovens (15 a 17 anos), que estudavam no período diurno e que não trabalhavam.

$\mathrm{Na}$ análise ajustada, com exceção da variável trabalho, que perdeu significância estatística, a idade dos adolescentes e o período de estudo continuaram associados à exposição a comportamentos de risco à saúde de forma simultânea, com maior probabilidade de os adolescentes mais velhos (18 a 19 anos) (17\%) e aqueles que estudavam no período noturno (28\%) apresentarem três ou mais comportamentos de risco, de forma simultânea, comparados aos seus pares mais jovens (15 a 17 anos) e que estudavam no período diurno, respectivamente (tabela 6).

\section{DISCUSSÃO}

O presente estudo avaliou diferentes comportamentos de risco à saúde de

TABELA 4. Razão de prevalência ajustada para comportamentos de risco em adolescentes escolares do ensino médio do Estado de Santa Catarina, Brasil, 2001

\begin{tabular}{|c|c|c|c|c|c|c|c|}
\hline Variável & $\begin{array}{c}\text { Níveis } \\
\text { insuficientes } \\
\text { de atividade } \\
\text { física } \\
\operatorname{RP}(\mathrm{IC} 95 \%)^{a}\end{array}$ & $\begin{array}{c}\text { Baixo } \\
\text { consumo de } \\
\text { frutas/verduras } \\
\text { RP }(\text { IC95\%) }\end{array}$ & $\begin{array}{c}\text { Tabagismo } \\
\text { RP (IC95\%) }\end{array}$ & $\begin{array}{l}\text { Consumo } \\
\text { abusivo de } \\
\text { bebidas } \\
\text { alcoólicas } \\
\text { RP (IC95\%) }\end{array}$ & $\begin{array}{l}\text { Consumo } \\
\text { de drogas } \\
\text { ilícitas } \\
\text { RP }(\text { IC95\%) }\end{array}$ & $\begin{array}{l}\text { Envolvimento } \\
\text { em brigas } \\
\operatorname{RP}(\mathrm{IC} 95 \%)^{\mathrm{a}}\end{array}$ & $\begin{array}{c}\text { Não utilizar } \\
\text { preservativos } \\
\text { com } \\
\text { regularidade } \\
\text { RP (IC95\%) }\end{array}$ \\
\hline \multicolumn{8}{|l|}{ Sexo } \\
\hline Feminino & 1 & 1 & 1 & 1 & 1 & 1 & 1 \\
\hline Masculino & $0,56(0,50$ a 0,62$)$ & $1,24(1,14$ a 1,35$)$ & $1,06(0,84$ a 1,32$)$ & $1,69(1,50$ a 1,90$)$ & $1,59(1,32$ a 1,90$)$ & $2,40(2,06$ a 2,79$)$ & $0,64(0,55$ a 074$)$ \\
\hline \multicolumn{8}{|l|}{ Idade (anos) } \\
\hline 15 a 17 & 1 & 1 & 1 & 1 & 1 & 1 & 1 \\
\hline 18 a 19 & $1,17(1,04$ a 1,31$)$ & $0,97(0,87$ a 1,08$)$ & $1,49(1,17$ a 1,90$)$ & $1,09(0,95$ a 1,25$)$ & $1,49(1,22$ a 1,82$)$ & $0,93(0,77$ a 1,11$)$ & $1,08(093$ a 1,25$)$ \\
\hline \multicolumn{8}{|c|}{ Região de residência } \\
\hline Rural & 1 & 1 & 1 & 1 & 1 & 1 & 1 \\
\hline Urbana & $0,95(0,84$ a 1,07$)$ & $1,15(1,01$ a 1,26$)$ & $2,28(1,59$ a 3,28$)$ & $0,93(0,80$ a 1,07$)$ & $2,62(1,91$ a 3,59$)$ & $1,20(0,97$ a 1,44$)$ & $1,13(0,92$ a 1,38$)$ \\
\hline \multicolumn{8}{|c|}{ Período de estudo } \\
\hline Diurno & 1 & & 1 & 1 & 1 & 1 & 1 \\
\hline Noturno & $1,36(1,23$ a 1,51$)$ & $1,02(0,93$ a 1,11$)$ & $1,52(1,19$ a 1,93$)$ & $1,31(1,15$ a 1,49$)$ & $1,28(1,05$ a 1,56$)$ & $0,97(0,86$ a 1,17$)$ & $1,14(0,98$ a 1,34$)$ \\
\hline \multicolumn{8}{|l|}{ Trabalho } \\
\hline Não & 1 & 1 & 1 & 1 & 1 & 1 & 1 \\
\hline Sim & $1,09(0,98$ a 1,20$)$ & $1,04(0,95$ a 1,14$)$ & $0,94(0,74$ a 1,18$)$ & $1,09(0,96$ a 1,23$)$ & $1,12(0,90$ a 1,33$)$ & $1,07(0,91$ a 1,24$)$ & $0,90(0,78$ a 1,05$)$ \\
\hline \multicolumn{8}{|c|}{ Renda familiar mensal } \\
\hline$\leq \mathrm{R} \$ 1000$ & 1 & 1 & 1 & 1 & 1 & 1 & 1 \\
\hline$>\mathrm{R} \$ 1000$ & $0,95(0,86$ a 1,06$)$ & $1,19(1,08$ a 1,31$)$ & $0,83(0,66$ a 1,05$)$ & $0,87(0,77$ a 1,01$)$ & $0,75(0,62$ a 0,90$)$ & $0,93(0,80$ a 1,09$)$ & $1,03(0,88$ a 1,20$)$ \\
\hline
\end{tabular}

\footnotetext{
a Razão de prevalência ajustada e respectivo intervalo de confiança de $95 \%$.
} 
FIGURA 1. Prevalência de comportamentos de risco à saúde de forma simultânea em adolescentes escolares do ensino médio do Estado de Santa Catarina, Brasil, 2001

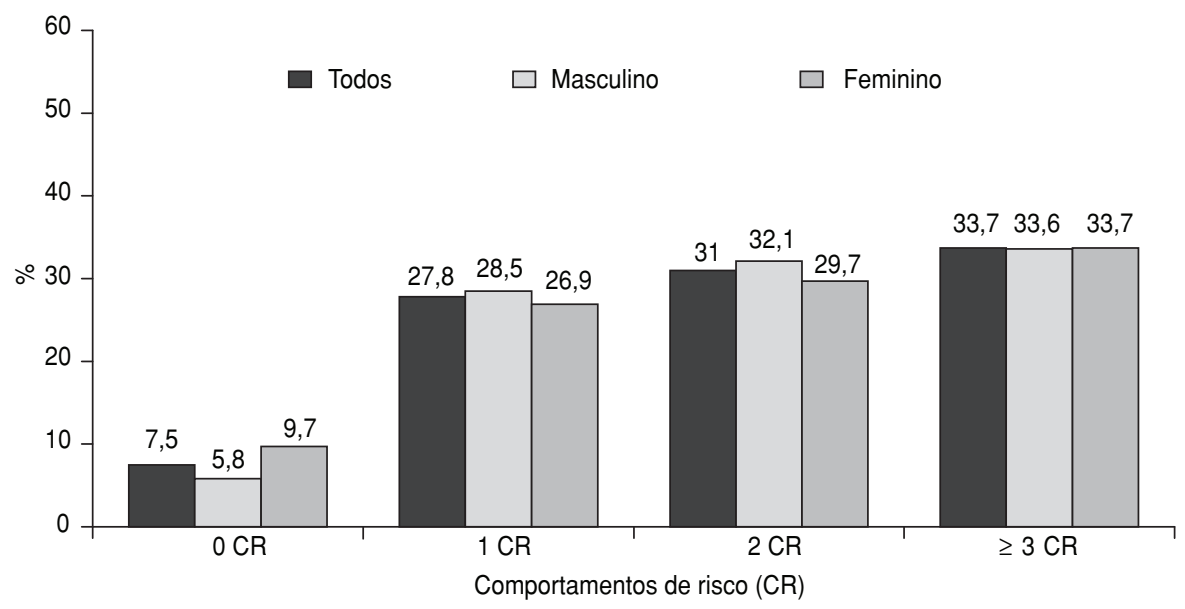

forma isolada e simultânea em adolescentes escolares do ensino médio da rede pública do Estado de Santa Catarina. Até onde sabemos, este é o primeiro estudo desenvolvido com base em uma amostra representativa de escolares do ensino médio da rede pública, de base estadual, que avaliou diferentes comportamentos de risco à saúde de forma isolada e simultânea.

Os resultados revelaram uma prevalência elevada de comportamentos de risco na população estudada e permitiram identificar cinco subgrupos de risco, a saber, adolescentes do sexo masculino, adolescentes de 18 a 19 anos de idade, adolescentes que residiam em área urbana, que estudavam no período noturno e que tinham maior renda familiar.

Com relação ao primeiro comportamento de risco avaliado (níveis insuficientes de atividade física), constatou-se que aproximadamente um terço $(36,5 \%)$ dos adolescentes foram classificados como insuficientemente ativos. Esses resultados são similares aos que foram observados em adolescentes da cidade de Pelotas, Região Sul do Brasil (20), e infe- riores aos encontrados em adolescentes norte-americanos (4).

Adolescentes do sexo feminino, adolescentes mais velhos (18 a 19 anos) e aqueles que estudavam no período noturno apresentaram menor envolvimento com prática de atividade física. O menor nível de prática de atividade física em adolescentes do sexo feminino em comparação com o sexo masculino tem sido amplamente descrito em estudos envolvendo adolescentes, independentemente da idade $(13,20,21)$ e do instrumento de medida utilizado (medida objetiva ou subjetiva) (22). Por outro lado, enquanto alguns estudos indicaram uma redução nos níveis de atividade física com o aumento da idade $(23,24)$, outros não confirmaram tal tendência $(25,26)$.

Assim como observado em estudos nacionais $(13,27)$ e internacionais $(4,28)$, os adolescentes do Estado de Santa Catarina também apresentaram baixo consumo de frutas/verduras: cerca de cinco em cada $10(46,5 \%)$ consumiam frutas/ verduras com frequência inferior a 4 dias por semana. O menor consumo de frutas/verduras entre rapazes, observado no presente estudo, já foi descrito anteriormente $(4,28)$, embora alguns estudos tenham apresentado achados distintos $(13,28)$.

A combinação de níveis reduzidos de prática de atividade física com baixo

TABELA 5. Prevalência e razão de prevalência bruta para comportamentos de risco de forma simultânea em adolescentes escolares do ensino médio do Estado de Santa Catarina, Brasil, 2001

\begin{tabular}{|c|c|c|c|c|c|c|c|c|c|}
\hline \multirow[b]{2}{*}{ Variável } & \multicolumn{3}{|c|}{$\begin{array}{c}\text { Modelo } 1 \\
0 \mathrm{CR}^{\mathrm{a}} \text { versus } \geq 1 \mathrm{CR}\end{array}$} & \multicolumn{3}{|c|}{$\begin{array}{c}\text { Modelo } 2 \\
1 \mathrm{CR} \text { versus } \geq 2 \mathrm{CR}\end{array}$} & \multicolumn{3}{|c|}{$\begin{array}{c}\text { Modelo } 3 \\
2 \mathrm{CR} \text { versus } \geq 3 \mathrm{CR}\end{array}$} \\
\hline & $\%^{b}$ & $\mathrm{RP}^{\mathrm{c}}$ & IC95\% & $\%^{b}$ & $\mathrm{RP}^{\mathrm{c}}$ & IC95\% & $\%^{b}$ & $\mathrm{RP}^{\mathrm{c}}$ & IC95\% \\
\hline \multicolumn{10}{|l|}{ Sexo } \\
\hline Feminino & 94,2 & 1 & & 65,7 & 1 & & 33,6 & 1 & \\
\hline Masculino & 90,3 & 0,95 & 0,89 a 1,02 & 63,4 & 0,96 & 0,88 a 1,05 & 33,7 & 1,01 & 0,89 a 1,12 \\
\hline \multicolumn{10}{|l|}{ Idade (anos) } \\
\hline 15 a 17 & 92,5 & 1 & & 64,1 & 1 & & 32,0 & 1 & \\
\hline 18 a 19 & 92,2 & 0,99 & 0,91 a 1,08 & 66,6 & 1,03 & 0,93 a 1,14 & $39,4^{d}$ & 1,23 & 1,07 a 1,40 \\
\hline \multicolumn{10}{|c|}{ Região de residência } \\
\hline Rural & 94,1 & 1 & & 61,5 & 1 & & 33,9 & 1 & \\
\hline Urbana & 92,0 & 0,97 & 0,89 a 1,06 & 65,4 & 1,06 & 0,95 a 1,18 & 33,6 & 0,99 & 0,85 a 1,15 \\
\hline \multicolumn{10}{|c|}{ Período de estudo } \\
\hline Diurno & 92,9 & 1 & & 61,4 & 1 & & 28,2 & 1 & \\
\hline Noturno & 92,1 & 0,99 & 0,92 a 1,06 & 67,2 & 1,09 & 0,98 a 1,19 & $37,9^{d}$ & 1,34 & 1,19 a 1,51 \\
\hline \multicolumn{10}{|l|}{ Trabalho } \\
\hline Não & 92,9 & 1 & & 62,9 & 1 & & 31,0 & 1 & \\
\hline Sim & 92,1 & 0,99 & 0,92 a 1,06 & 66,6 & 1,07 & 0,98 a 1,16 & $35,7^{d}$ & 1,15 & 1,02 a 1,29 \\
\hline \multicolumn{10}{|c|}{ Renda familiar mensal } \\
\hline$\leq \mathrm{R} \$ 1000$ & 93,4 & 1 & & 64,8 & 1 & & 33,9 & 1 & \\
\hline$>\mathrm{R} \$ 1000$ & 90,6 & 1,03 & 0,95 a 1,11 & 64,5 & 0,99 & 0,91 a 1,09 & 33,1 & 1,02 & 0,89 a 1,16 \\
\hline
\end{tabular}

a Comportamento de risco.

${ }^{b}$ Prevalência de comportamentos de risco simultâneos.

${ }^{c}$ Razão de prevalência bruta.

$d$ Teste para heterogeneidade, $p<0,01$. 
TABELA 6. Razão de prevalência ajustada para comportamentos de risco de forma simultânea em adolescentes escolares do ensino médio do Estado de Santa Catarina, Brasil, 2001

\begin{tabular}{|c|c|c|c|c|c|c|}
\hline \multirow[b]{2}{*}{ Variável } & \multicolumn{2}{|c|}{$\begin{array}{c}\text { Modelo } 1 \\
0 \mathrm{CR}^{\mathrm{a}} \text { versus } \geq 1 \mathrm{CR}\end{array}$} & \multicolumn{2}{|c|}{$\begin{array}{c}\text { Modelo } 2 \\
1 \mathrm{CR} \text { versus } \geq 2 \mathrm{CR}\end{array}$} & \multicolumn{2}{|c|}{$\begin{array}{c}\text { Modelo } 3 \\
2 \mathrm{CR} \text { versus } \geq 3 \mathrm{CR}\end{array}$} \\
\hline & $\begin{array}{c}\mathrm{RP} \\
\text { ajustada }^{\mathrm{b}}\end{array}$ & IC95\% & $\begin{array}{c}\mathrm{RP} \\
\text { ajustada }^{\mathrm{b}}\end{array}$ & IC95\% & $\begin{array}{c}\mathrm{RP} \\
\text { ajustada }^{\mathrm{b}}\end{array}$ & IC95\% \\
\hline \multicolumn{7}{|l|}{ Sexo } \\
\hline Feminino & 1 & & 1 & & 1 & \\
\hline Masculino & 0,95 & 0,89 a 1,03 & 0,95 & 0,87 a 1,04 & 0,96 & 0,85 a 1,08 \\
\hline \multicolumn{7}{|l|}{ Idade (anos) } \\
\hline 15 a 17 & 1 & & 1 & & 1 & \\
\hline 18 a 19 & 1,00 & 0,91 a 1,09 & 1,01 & 0,91 a 1,13 & 1,17 & 1,01 a 1,34 \\
\hline \multicolumn{7}{|c|}{ Região de residência } \\
\hline Rural & 1 & & 1 & 1 & 1 & \\
\hline Urbana & 0,97 & 0,89 a 1,07 & 1,05 & 0,94 a 1,17 & 0,98 & 0,84 a 1,14 \\
\hline \multicolumn{7}{|c|}{ Período de estudo } \\
\hline Diurno & 1 & & 1 & & 1 & \\
\hline Noturno & 0,99 & 0,92 a 1,07 & 1,08 & 0,98 a 1,18 & 1,28 & 1,13 a 1,46 \\
\hline \multicolumn{7}{|l|}{ Trabalho } \\
\hline Não & 1 & & 1 & & 1 & \\
\hline Sim & 1,00 & 0,92 a 1,08 & 1,05 & 0,96 a 1,15 & 1,06 & 0,93 a 1,20 \\
\hline \multicolumn{7}{|c|}{ Renda familiar mensal } \\
\hline$\leq \mathrm{R} \$ 1000$ & 1 & & 1 & & 1 & \\
\hline$>\mathrm{R} \$ 1000$ & 1,02 & 0,94 a 1,11 & 1,00 & 0,91 a 1,10 & 0,99 & 0,86 a 1,13 \\
\hline
\end{tabular}

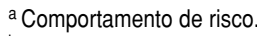

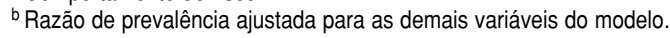

consumo de frutas/verduras, observada neste estudo, pode contribuir para o aumento da prevalência de sobrepeso/obesidade na população jovem. Um em cada 10 jovens deste estudo ( $12,7 \%$ nos rapazes e $8,1 \%$ nas moças) apresentou excesso de peso corporal (dados não apresentados em tabela).

Menos de $10 \%$ dos jovens declararam fumar, possivelmente um reflexo das constantes campanhas e ações direcionadas ao controle do tabaco no Brasil (29). Um estudo de base populacional realizado com adolescentes (15 a 18 anos) na cidade de Pelotas, Região Sul do Brasil, encontrou prevalência de $16,6 \%$ de tabagismo (fumar pelo menos uma vez por semana no último mês), com índices mais elevados em adolescentes do sexo feminino (14). Por outro lado, assim como observado em outras investigações (4, 30-32), no presente estudo, a prevalência de tabagismo foi similar entre rapazes e moças, porém mais elevada nos jovens mais velhos $(14,31-34)$ e nos que residiam em área urbana (34).

O consumo abusivo de bebidas alcoólicas foi referido por $27,4 \%$ dos adolescentes. Esses resultados são similares aos observados em adolescentes da cidade de Florianópolis, Estado de Santa Catarina (13), e inferiores aos encontrados em adolescentes da cidade de Pelotas, Estado do Rio Grande do Sul (14), e em norte-americanos (4). Adolescentes do sexo masculino e que estudavam no período noturno apresentaram maior prevalência de uso abusivo de bebidas alcoólicas em comparação com as moças e os adolescentes que estudavam no período diurno, respectivamente. Esses resultados se assemelham aos encontrados em outros estudos realizados com adolescentes brasileiros $(13,14)$ e norte-americanos (4), que também relataram maior consumo de bebidas alcoólicas em rapazes. Contrariando resultados de alguns estudos (35) e reforçando achados de outros $(34,36)$, o consumo abusivo de bebidas alcoólicas não diferiu entre jovens que residiam em área urbana e rural. Tais divergências podem decorrer de características sociodemográficas e culturais específicas de cada amostra, assim como de diferentes formas de medir e classificar esse comportamento (37).

Um em cada 10 adolescentes referiu já ter feito uso de algum tipo de droga ilícita, com maior frequência entre os rapazes, nos adolescentes mais velhos (18 a 19 anos), nos que residiam em área urbana e nos que estudavam no período noturno. Esses resultados reforçam achados de estudos prévios $(12,14,32)$.

Cerca de dois em cada 10 adolescentes $(16,5 \%)$ referiram envolvimento em brigas, sendo esta uma prática mais frequente entre os rapazes em comparação às moças. Tais resultados são similares aos descritos em outros estudos realiza- dos com adolescentes $(4,12)$, que relataram 15 a $35 \%$ de adolescentes envolvidos em brigas nos últimos 12 meses, na maioria rapazes.

Entre os adolescentes que tinham vida sexualmente ativa, 38,3\% referiram não utilizar preservativos regularmente nas relações sexuais, principalmente adolescentes do sexo feminino. A prevalência elevada do uso irregular de preservativos entre adolescentes, estimada em 30 a $40 \%(4,12)$, tem sido amplamente demonstrada em estudos internacionais (4, $8,38)$ e nacionais (15-17). Tal comportamento expõe esses jovens a doenças sexualmente transmissíveis e a episódios de gravidez cada vez mais precoces. Dados da Pesquisa Nacional por Amostra de Domicílios (PNAD) revelaram que, em 2005, 19,9\% dos nascidos vivos foram filhos de mães adolescentes (39).

Ao considerar a exposição simultânea a diferentes comportamentos de risco à saúde, verificou-se que $64,7 \%$ dos adolescentes estavam expostos a dois ou mais comportamentos de risco. Os adolescentes mais velhos (18 a 19 anos) e os que estudavam no período noturno apresentaram maior probabilidade (cerca de 20 a $30 \%$ ) de exposição simultânea a três ou mais comportamentos de risco à saúde em comparação aos seus pares mais jovens (15 a 17 anos) e aos que estudavam no período diurno (manhã/tarde).

A prevalência elevada de exposição simultânea a diferentes comportamentos de risco em adolescentes tem sido descrita em estudos nacionais (13) e internacionais $(40,41)$. A combinação de parâmetros de risco no estilo de vida dos adolescentes associa-se aos baixos níveis de qualidade de vida (1), à presença de doenças sexualmente transmissíveis e de gravidez indesejada $(32,38)$, a tentativas de suicídio (32), a acidentes por veículos a motor e por armas de fogo, ao envolvimento em brigas violentas (8) e ao baixo desempenho escolar (42). A exposição precoce e prolongada a alguns desses comportamentos de risco (níveis insuficientes de atividade física, baixo consumo de frutas/verduras, tabagismo e consumo abusivo de bebidas alcoólicas) está associada à presença de fatores de risco para doença cardiovascular $(10,43)$ e à presença de doenças cardiovasculares na adolescência e idade adulta $(44,45)$.

Algumas limitações deste estudo devem ser destacadas. Por se tratar de um estudo de base escolar, realizado na rede pública, a generalização dos resultados para todos 
os adolescentes de 15 a 19 anos de idade do Estado de Santa Catarina fica comprometida. Não foi possível coletar dados dos escolares que não estavam em sala de aula no dia da coleta, dos que haviam desistido do ano escolar e daqueles que estavam matriculados em escolas privadas, não se podendo, portanto, descartar um possível viés de seleção.

A utilização de questionários para medir atividade física é outra limitação. Apesar de esses instrumentos apresentarem bons níveis de reprodutibilidade, sua validade é moderada, já que os adolescentes tendem a superestimar sua participação em atividades físicas vigorosas e subestimar sua participação em atividades físicas moderadas (22).

Finalmente, mesmo que o questionário tenha sido anônimo, não se pode des- cartar a possibilidade de viés de informação em relação a consumo abusivo de bebidas alcoólicas, tabagismo e, especialmente, consumo de drogas ilícitas e nãoutilização regular de preservativos nas relações sexuais. É possível supor, portanto, que tais prevalências sejam superiores às relatadas.

Os resultados do presente estudo permitem concluir que há uma elevada proporção de adolescentes com níveis insuficientes de atividade física, hábitos alimentares inadequados (baixo consumo de frutas/verduras) e atividade sexual sem o uso regular de preservativos. Destaca-se a importância da identificação dos subgrupos de risco proporcionada pelo estudo. Finalmente, a exposição simultânea a vários comportamentos de risco observada em adolescentes de ambos os sexos e nos demais estratos demográficos e socioeconômicos é outro resultado importante, com pouco mais de sete em cada 10 adolescentes expostos a dois ou mais comportamentos de risco à saúde.

Na perspectiva da saúde pública, os dados aqui apresentados podem contribuir para subsidiar o desenvolvimento de programas de promoção da saúde no ambiente escolar, principalmente no que diz respeito aos subgrupos de risco identificados.

Agradecimento. Os autores agradecem aos estudantes, professores e administradores das escolas envolvidas neste estudo, bem como à Secretaria de Estado da Educação de Santa Catarina. Este estudo foi financiado pelo Conselho Nacional de Desenvolvimento Científico e Tecnológico (CNPq).

\section{REFERÊNCIAS}

1. Topolski TD, Patrick DL, Edwards TC, Huebner CE, Connell FA, Mount KK. Quality of life and health-risk behaviors among adolescents. J Adolesc Health. 2001;29(6):426-35.

2. Zahran HS, Zack MM, Vernon-Smiley ME, Hertz MF. Health-related quality of life and behaviors risky to health among adults aged 18-24 years in secondary or higher education-United States, 2003-2005. J Adolesc Health. 2007;41(4):389-97.

3. Currie C, Roberts C, Morgan A, Smith R, Settertobulte W, Samdal O, et al. Young people's health in context. Health Behavior in Schoolaged Children (HBSC) study: international report from the 2001/2002 survey. Copenhagen: World Health Organization; 2004. (Health Policy for Children and Adolescents, No. 4.) Disponível em: http://www.euro. who.int/eprise/main/who/information sources/publications/catalogue/20040518_1. Acessado em 10 de abril de 2005.

4. Centers for Disease Control and Prevention. Youth risk behavior surveillance - United States, 2005. MMWR. 2006;55(SS-5). Disponível em: http://www.cdc.gov/mmwr/ PDF/SS/SS5505.pdf. Acessado em 7 de junho de 2007.

5. Barros MDA, Ximenes R, Lima MLC. Mortalidade por causas externas em crianças e adolescentes: tendências de 1979 a 1995. Rev Saude Publica. 2001;35(2):142-9.

6. Brasil, Ministério da Saúde. Saúde Brasil 2004 - uma análise da situação de saúde. Brasília: Ministério da Saúde; 2004.

7. Díez JMB, Pérez BA, Forcadell PP, Peñacoba RM, Sabaté MN, Boronat AA. Associación entre la agrupación (clustering) de factores de riesgo cardiovascular y el riesgo de enfermedad cardiovascular. Rev Esp Salud Publica. 2002;76(1):7-15.

8. Xing Y, Ji C, Zhang L. Relationship of binge drinking and other health-compromising behaviors among urban adolescents in China. J Adolesc Health. 2006;39(4):495-500.
9. Paavola M, Vartiainen E, Haukkala A. Smoking, alcohol use, and physical activity: a 13year longitudinal study ranging from adolescence into adulthood. J Adolesc Health. 2004; 35(3):238-44.

10. Twisk JWR, Mechelen WV, Kemper HCG, Post GB. The relation between "long-term ex-

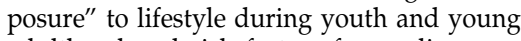
adulthood and risk factors for cardiovascular disease at adult age. J Adolesc Health. 1997;20(4):309-19.

11. Guedes DP, Guedes JERP, Barbosa DS, Oliveira JA, Stanganelli LCR. Fatores de risco cardiovasculares em adolescentes: indicadores biológicos e comportamentais. Arq Bras Cardiol. 2006;86(6):439-50

12. Carlini-Cotrim B, Gazal-Carvalho C, Gouveia $\mathrm{N}$. Comportamentos de risco entre jovens estudantes das redes pública e privada da área metropolitana do estado de São Paulo. Rev Saude Publica. 2000;34(6):636-45.

13. Farias Júnior J, Lopes A. Comportamentos de risco à saúde em adolescentes. Rev Bras Cienc Mov. 2004;12(1):7-12. Disponível em: http:// www.ucb.br/mestradoef/RBCM/12/12\%20\%201/c_12_1_1.pdf . Acessado em 12 de outubro de 2004.

14. Horta RL, Horta BL, Pinheiro RT, Morales B, Strey MN. Tabaco, álcool e outras drogas entre adolescentes em Pelotas, Rio Grande do Sul, Brasil: uma perspectiva de gênero. Cad Saude Publica. 2007;23(4):775-83.

15. Miranda AE, Gadelha AMJ, Szwarcwald CL. Padrão de comportamento relacionado às práticas sexuais e ao uso de drogas de adolescentes do sexo feminino residentes em Vitória, Espírito Santo, Brasil, 2002. Cad Saude Publica. 2005;21(1):207-16.

16. Almeida MCC, Aquino EML, Barros AP. School trajectory and teenage pregnancy in three Brazilian state capitals. Cad Saude Publica. 2006;22(7):1397-409.

17. Rocha CLA, Horta BL, Pinheiro RT, Cruzeiro ALS, Cruz S. Use of contraceptive methods by sexually active teenagers in Pelotas, Rio Grande do Sul State, Brazil. Cad Saude Publica. 2007;23(12):2862-8.

18. Instituto Brasileiro de Geografia e Estatística. Censo Demográfico 2000. Rio de Janeiro: IBGE; 2002. Disponível em: http://www. ibge.gov.br/home/estatistica/populacao/ce nso2000/default.shtm. Acessado em 8 de maio de 2007.

19. Estado de Santa Catarina, Secretaria de Estado da Educação e do Desporto, Diretoria de Planejamento e Coordenação, Gerência de Estatística e Informática. Censo escolar. Florianópolis: Secretaria de Estado da Educação e do Desporto; 2001.

20. Oehlschlaeger MHK, Pinheiro RT, Horta B, Gelatti C, Sant'Ana P. Prevalência e fatores de associados ao sedentarismo em adolescentes de área urbana. Rev Saude Publica. 2004; 38(2):157-63.

21. Varo JJ, Martínez-González MA, De IralaEstévez J, Kearney J, Gibney M, Martinez JA. Distribution and determinants of sedentary lifestyles in the European Union. Int J Epidemiol. 2003;32(1):138-46.

22. Troped PJ, Wiecha JL, Fragala MS, Matthews CE, Finkelstein DM, Kim J, et al. Reliability and validity of YRBS physical activity items among middle school students. Med Sci Sports Exerc. 2007;39(3):416-25.

23. Telama R, Yang X. Decline of physical activity from youth to young adulthood in Finland. Med Sci Sports Exerc. 2000;32(9):1617-22.

24. Van Mechelen W, Twisk JWR, Post GB, Snel J, Kemper HCG. Physical activity of young people: the Amsterdam longitudinal growth and health study. Med Sci Sports Exerc. 2000; 32(9):1610-6.

25. Cantera-Garde MA, Devís-Devís J. Physical activity levels of secondary school Spanish adolescents. Eur J Physical Education. 2000;5(1): $28-44$.

26. Guedes DP, Guedes JERP, Barbosa DS, Oliveira JA. Níveis de prática de atividade 
física habitual em adolescentes. Rev Bras Med Esporte. 2001;7(6):187-200.

27. Toral N, Slater B, Cintra IP, Fisberg M. Comportamento alimentar de adolescentes em relação ao consumo de frutas e verduras. Rev Nutr. 2006;19(3):331-40.

28. Cavadini C, Decarli B, Dirren H, Cauderay M, Narring F, Michaud P. Assessment of adolescent food habits in Switzerland. Appetite. 1999;32(1):97-106.

29. Cavalcante TM. O controle do tabagismo no Brasil: avanços e desafios. Rev Psiquiatr Clin. 2005;32(5):283-300.

30. Malcon MC, Menezes AMB, Maia MF, Chatkin M, Victora CG. Prevalência e fatores de risco para tabagismo em adolescentes na América do Sul: uma revisão sistemática da literatura. Rev Panam Salud Publica. 2003; 134(4):222-8.

31. Azevedo A, Machado AP, Barros H. Tobacco smoking among Portuguese high-school students. Bull World Health Organ. 1999;77(6): 509-14.

32. Anteghini $\mathrm{M}$, Fonseca $\mathrm{H}$, Ireland $\mathrm{M}$, Blum RW. Health risk behaviors and associated risk and protective factors among Brazilian adolescents in Santos, Brazil. J Adolesc Health. 2001;28(4):295-302.

33. Malcon MC, Menezes AM, Chakin M. Prevalência e fatores de risco para tabagismo em adolescentes. Rev Saude Publica. 2003; 37(1):1-7.
34. Yamamoto-Kimura L, Posadas-Romero C, Posadas-Sánchez R, Zamora-González J, Cardoso-Saldaña G, Ramírez I. Prevalence and interrelations of cardiovascular risk factors in urban and rural Mexican adolescents. J Adolesc Health. 2006;38(5):591-8.

35. Zhou X, Sub Z, Denga H, Xianga X, Chen $\mathrm{H}$, Haoa W. A comparative survey on alcohol and tobacco use in urban and rural populations in the Huaihua District of Hunan Province, China. Alcohol. 2006;39(2): 87-96.

36. Kelishadi R, Sadri G, Tavasoli AA, Kahbazi M, Roohafza HR, Sadeghi M, et al. A prevalência cumulativa de fatores de risco para doença cardiovascular em adolescentes iranianos - IHHP-HHPC. J Pedriatr (Rio J). 2005; 81(6):447-53.

37. Brady JE, Weitzmanb BC. Inconsistencies in place definition: How different operational place definitions affect estimates of adolescent smoking and drinking risk. Health Place. 2007;13(2):562-8.

38. Hidalgo I, Garrido G, Hernandez M. Health status and risk behavior of adolescents in the north of Madrid, Spain. J Adolesc Health. 2000;27(5):351-60.

39. Instituto Brasileiro de Geografia e Estatística. Pesquisa Nacional por Amostra de Domicílios. Rio de Janeiro: IBGE; 2005. Disponível em: http://www.ibge.gov.br/home/estatistica/ populacao/trabalhoerendimento/pnad2005/ default.shtm. Acessado em 29 de março de 2006.

40. Felton GM, Pate RR, Parsons MA, Ward DS, Saunders RP, Trost S, et al. Health risk behaviors of rural sixth graders. Res Nurs Health. 1998;21(6):474-85.

41. Ohene S-A, Ireland M, Blum R. The clustering of risk behaviors among Caribbean youth Matern Child Health J. 2005;9(1):91-100.

42. Tavares BT, Béria JU, Lima MS. Prevalência do uso de drogas e desempenho escolar entre adolescentes. Rev Saude Publica. 2001;35(2): 150-8.

43. Twisk JW, Kemper HC, Van Mechelen W, Post GB. Clustering of risk factors for coronary heart disease: the longitudinal relationship with lifestyle. Ann Epidemiol. 2001; 11(3):157-65.

44. Berenson GS, Srnivasan SR. Cardiovascular risk factors in youth with implications for aging: the Bogalusa Heart Study. Neurobiol Aging. 2005;26(3):303-7.

45. Raitakari OT, Juonala M, Kähönen M, Taittonen L, Laitinen T, Mäki-Torkko N, et al. Cardiovascular risk factors in childhood and carotid artery intima-media thickness in adulthood. The Cardiovascular Risk in Young Finns Study. JAMA. 2003;290(17): 2277-83.

Manuscrito recebido em 1 de dezembro de 2007. Aceito em versão revisada em 15 de abril de 2008 .

ABSTRACT Objective. To determine the prevalence of health risk behaviors and to analyze factors associated with exposure to such behaviors among adolescents in the state of Santa Catarina in the south of Brazil.

Health risk behaviors among adolescents in the south of Brazil: prevalence and associated factors

Methods. Adolescents attending 240 high school classes at the state's public schools answered a questionnaire that collected demographics (sex, age, area of residence, and day versus night classes), social and economic data (working status and monthly family income), and information on health risk behaviors (insufficient levels of physical activity, low intake of fruits/vegetables, smoking, alcohol abuse, illicit drug use, involvement in physical fights, and irregular use of condoms).

Results. The completed questionnaires of 5028 adolescents (2 984 females and 2044 males), $15-19$ years of age (mean $=17.0$; standard deviation $=1.12$, were considered valid. The most prevalent health risk behaviors were insufficient levels of physical activity (36.5\%), low intake of fruits/vegetables (46.5\%), and irregular use of condoms (38.3\%). Approximately 7 of 10 adolescents $(64.7 \%)$ were exposed to two or more risk behaviors simultaneously. The following risk subgroups were identified: male adolescents, adolescents from 18-19 years of age, living in urban areas, studying at night, and having a higher family income.

Conclusions. The percentage of adolescents exposed to health risk behaviors was high, especially when simultaneous exposure to different behaviors was considered. These results can contribute to developing health promotion campaigns for the school setting that are specifically aimed at the risk subgroups identified.

Key words Adolescent behavior, risk-taking, students, life style, health, Brazil. 